\title{
In Vivo Efficacy of the Combination of Ciprofloxacin and Cefotaxime against Vibrio vulnificus Sepsis
}

\author{
Hee-Chang Jang ${ }^{1,2}$, Su-Mi Choi ${ }^{1}$, Hee Kyung Kim ${ }^{1}$, Sung-Eun Kim ${ }^{1}$, Seung-Ji Kang ${ }^{1}$, Kyung-Hwa Park ${ }^{1}$, \\ Phil Youl Ryu ${ }^{3}$, Tae-Hoon Lee ${ }^{4}$, Young Ran Kim ${ }^{5}$, Joon Haeng Rhee ${ }^{2,3}$, Sook-In Jung ${ }^{1 * 9}$, Hyon E Choy ${ }^{2,3 * 9}$
}

1 Department of Infectious diseases, Chonnam National University Medical School, Gwang-ju, Republic of Korea, 2 Research Institute of Vibrio Infection and Genome Research Center for Enteropathogenic Bacteria, Gwang-ju, Republic of Korea, 3 Microbiology, Chonnam National University Medical School, Gwang-ju, Republic of Korea, 4 Department of Biochemistry, School of Dentistry, Chonnam National University, Gwang-ju, Republic of Korea, 5 Department of Pharmaceutical Engineering, Dongshin University, Naju, Republic of Korea

\begin{abstract}
Objectives: The in vivo efficacy of a cefotaxime-ciprofloxacin combination against Vibrio vulnificus and the effects on rtxA1 expression of commonly used antibiotics are unknown.

Methods: In vitro time-kill studies were performed to evaluate synergism. Female BALB/c mice were injected subcutaneously with $1 \times 10^{7}$ or $1 \times 10^{8}$ cfu of $V$. vulnificus. Antibiotic therapy was initiated at $2 \mathrm{~h}$ after inoculation in the following four therapy groups: cefotaxime; ciprofloxacin; cefotaxime-plus-ciprofloxacin; and cefotaxime-plus-minocycline. The cytotoxicity of $V$. vulnificus for HeLa cells was measured using the lactate dehydrogenase assay; $r$ txA 1 transcription was measured in a transcriptional reporter strain using a $\beta$-galactosidase assay.

Results: In vitro time-kill assays exhibited synergism between cefotaxime and ciprofloxacin. In the animal experiments, the 96-h survival rate for the cefotaxime-plus-ciprofloxacin group $(85 \% ; 17 / 20)$ was significantly higher than that of the cefotaxime-plus-minocycline $(35 \% ; 7 / 20)$ and cefotaxime alone $(0 \% ; 0 / 20)$ groups $(P<0.05$ for both). Bacterial counts in the liver and spleen were significantly lower in the cefotaxime-plus-ciprofloxacin group 24 and $48 \mathrm{~h}$ after treatment, relative to the other groups. At sub-inhibitory concentrations, ciprofloxacin inhibited more effectively $r t x A 1$ transcription and mammalian cell cytotoxicity than either minocycline or cefotaxime $(P<0.05$ for both).

Conclusions: Ciprofloxacin is more effective at reducing rtxA1 transcription and subsequent cytotoxicity than either minocycline or cefotaxime, and the combination of ciprofloxacin and cefotaxime was more effective in clearing $V$. vulnificus in vivo than previously used regimens. These data suggest that the combination of ciprofloxacin and cefotaxime is an effective option for the treatment of $V$. vulnificus sepsis in humans.
\end{abstract}

Citation: Jang H-C, Choi S-M, Kim HK, Kim S-E, Kang S-J, et al. (2014) In Vivo Efficacy of the Combination of Ciprofloxacin and Cefotaxime against Vibrio vulnificus Sepsis. PLoS ONE 9(6): e101118. doi:10.1371/journal.pone.0101118

Editor: Lars Kaderali, Technische Universität Dresden, Medical Faculty, Germany

Received February 19, 2014; Accepted June 3, 2014; Published June 30, 2014

Copyright: (c) 2014 Jang et al. This is an open-access article distributed under the terms of the Creative Commons Attribution License, which permits unrestricted use, distribution, and reproduction in any medium, provided the original author and source are credited.

Funding: This work was supported by a grant from the Research Institute of Medical Sciences, Chonnam National University (2011-CURIMS-DR005). The funder had no role in study design, data collection and analysis, decision to publish, or preparation of the manuscript.

Competing Interests: The authors have declared that no competing interests exist.

*Email: sijung@chonnam.ac.kr (SIJ); hyonchoy@chonnam.ac.kr (HEC)

9 These authors contributed equally to this work.

\section{Introduction}

Vibrio vulnificus is an opportunistic human pathogen that causes rapidly fatal sepsis, as well as skin and soft tissue infections, including necrotizing fasciitis. This pathogen is transmitted to humans through the exposure of wounds to seawater or the ingestion of seafood, and causes septicemia, especially in patients with immunosuppression and iron overload, as is the case in chronic liver disease [1,2]. The mortality rate for $V$. vulnificus septicemia is $\sim 70 \%[1]$, and the major virulence factors of this pathogen include capsular polysaccharides, exotoxins such as the repeats-in-toxin Al (RtxAl), hemolysin, metalloproteinase, and iron acquisition systems. Among these, RtxAl is a potent cytotoxic virulence factor that plays important roles in the pathogenesis and lethality of $V$. vulnificus infections [3-10].
Immediate surgical intervention and the administration of effective antibiotics are essential to reduce the mortality rate for $V$. vulnificus sepsis $[1,2,11]$. Antibiotics that are known to be effective include beta-lactams, tetracyclines, and quinolones. Among these, the combinations of a third-generation cephalosporin with tetracycline or quinolone monotherapy are most often recommended, based on the results of in vitro [12] and in vivo research $[13,14]$ and several retrospective clinical studies $[15,16]$. However, the mortality rates remain high, suggesting the need for adjuvant therapies $[17,18]$ and more effective antibiotic regimens.

A combination of cefotaxime and a quinolone has a synergistic effect on enteric Gram-negative pathogens, including Escherichia coli [19] and Salmonella species [20,21]. It has also been shown that the combination of cefotaxime and ciprofloxacin has synergism and superior in vitro bactericidal activity against $V$. vulnificus than 
currently recommended regimens, such as ciprofloxacin alone or cefotaxime plus doxycycline [22]. However, the in vivo efficacy of this combination remains unclear. Moreover, although RtxAl plays a key role in the virulence of $V$. vulnificus and mortality in $V$. vulnificus-related sepsis, the effects of commonly used therapeutic antibiotics at sub-inhibitory concentrations on rtxA1 expression have not been evaluated. To address these issues, we evaluated the in vitro synergism and in vivo efficacy of the combination of ciprofloxacin and cefotaxime against $V$. vulnificus sepsis, as well as the effect of commonly used antibiotics on $r t x A 1$ expression.

\section{Materials and Methods}

\section{Ethics}

All animal experiments were carried out in accordance with the guidelines set forth by the Institutional Animal Care and Use Committee (IACUC) of Chonnam National University and the guidelines for animal experiments set forth by the Korean Food and Drug Administration (KFDA) [23]. The study protocol was approved by the IACUC of Chonnam National University Hwasun Hospital.

\section{Bacterial strains and in vitro time-kill assay}

V. vulnificus CMCP6, which is a clinical isolate from Chonnam National University Hospital for which full genome sequence is available (GenBank accession nos. AEO16795 and AEO16796) [24], was used in the time-kill study and animal infection study. $V$. vulnificus strains MO6-24/O and CMM770 (MO6-24/O background with a deletion mutation in the $r t x A 1$ gene) [25] were used in the cytotoxicity assay and transcriptional reporter assay.

The minimal inhibitory concentrations (MICs) of cefotaxime, minocycline, and ciprofloxacin were determined by the microdilution method according to the guidelines of the Clinical and Laboratory Standards Institute [26]. In vitro time-kill studies were performed to evaluate synergy, as described previously [22]. Cefotaxime (Chong Kun Dang Pharmaceutical, Seoul, Republic of Korea), minocycline (Sigma-Aldrich, St. Louis, MO) and ciprofloxacin (Ildong Pharmaceutical, Seoul, Republic of Korea) were used throughout the study. Synergy was defined as a $\geq 2-$ $\log _{10} \mathrm{cfu} / \mathrm{mL}$ increase in killing at $24 \mathrm{~h}$ using the combination therapy compared with the level of killing achieved with the most active single drug.

\section{In vivo animal study}

Female, specific pathogen free, 8-week-old BALB/c mice (Samtako, Osan, Republic of Korea) with an average weight of $20 \mathrm{~g}$ were used throughout the study. The inocula were prepared as described previously [14]. We chose $1 \times 10^{7}$ and $1 \times 10^{8} \mathrm{cfu}$ as the initial inocula, based on previous studies $[12,14]$ and our preliminary results. To induce an iron-overload status, which increases susceptibility to $V$. vulnificus and more closely represents the iron-overloaded condition seen clinically, $900 \mu \mathrm{g}$ ferric ammonium citrate was administered intra-peritoneally (i.p.) $30 \mathrm{~min}$ before $V$. vulnificus inoculation [27]. Next, $1 \times 10^{7}$ or $1 \times 10^{8}$ cfu $V$. vulnificus were injected subcutaneously into the area over the right thigh $[12,14]$.

Each experiment consisted of five groups: a control group and groups treated with cefotaxime, ciprofloxacin, cefotaxime-plusciprofloxacin, and cefotaxime-plus-minocycline. All antibiotics were initially given i.p. beginning $2 \mathrm{~h}$ after the animal was infected. Cefotaxime $(30 \mathrm{mg} / \mathrm{kg}$ body weight [BW] i.p.) was given every $6 \mathrm{~h}$. Minocycline (loading dose of $4 \mathrm{mg} / \mathrm{kg} \mathrm{BW}$, followed by a maintenance dose of $2 \mathrm{mg} / \mathrm{kg}$ BW i.p.) was given every $12 \mathrm{~h}$. Ciprofloxacin (8 mg/kg BW, i.p.) was given every $12 \mathrm{~h}$, as described previously $[12,14]$. Control mice received $0.1 \mathrm{~mL}$ sterile saline every $6 \mathrm{~h}$. Antibiotics were given for a total of $42 \mathrm{~h}$. The condition of animals was monitored every 6 h. Humane endpoints were used during the survival study; animals were euthanized using ether when they exhibited a combined clinical criteria $(\geq 8$ points), according to KFDA guidelines [23]. Clinical endpoints were defined using the following scoring system: change in body weight, $0-3$ points; hair coat, $0-2$ points; eye opening, $0-2$ points; activity, $0-2$ points; posture $0-3$ points.

In addition, we counted the numbers of viable bacteria in the livers and spleens 24 and $48 \mathrm{~h}$ of infected mice after initiation of antibiotic treatment using an initial inoculum of $1 \times 10^{7} \mathrm{cfu}$. Mice were humanely euthanized using ether at 24 and $48 \mathrm{~h}$, and the livers and spleens were homogenized. Then, the homogenized tissues were serially diluted and plated onto Brain-Heart infusion agar, to quantify the bacteria.

\section{Effects of sub-inhibitory concentrations of antibiotics on V. vulnificus cytotoxicity for HeLa cells}

V. vulnificus cytotoxicity for HeLa cells was measured using the CytoTox96 non-radioactive cytotoxicity assay kit (Promega,

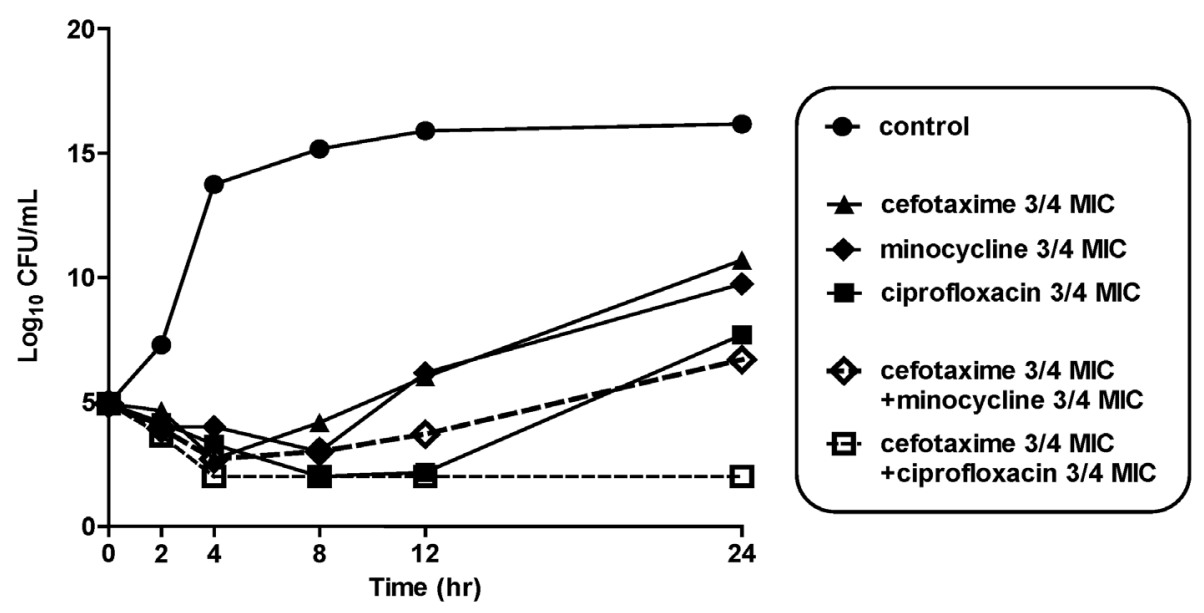

Figure 1. Time-kill curves for V. vulnificus CMCP6 after incubation with 3/4 MICs of cefotaxime alone, minocycline alone, ciprofloxacin alone, cefotaxime-plus-ciprofloxacin or cefotaxime-plus-minocycline. CFU, colony-forming unit; MIC, minimum inhibitory concentration.

doi:10.1371/journal.pone.0101118.g001 


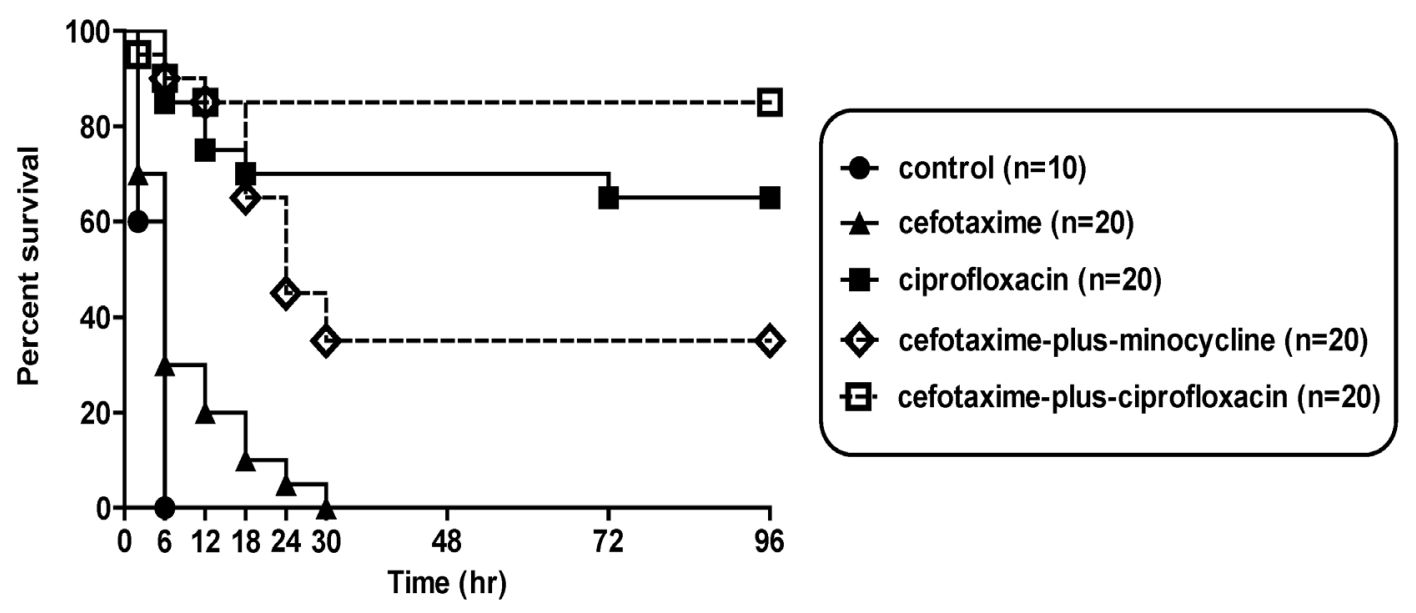

Figure 2. Survival rates of mice in each treatment group inoculated with $1 \times 10^{8}$ cfu $\mathbf{V}$. vulnificus. The $96-\mathrm{h}$ survival rate of the cefotaximeplus-ciprofloxacin group $(85 \%, 17 / 20)$ was significantly higher than that of the cefotaxime $(0 \%, 0 / 20)$ or the cefotaxime-plus-minocycline groups $(35 \%, 7 / 20)(P<0.001$ and $P=0.003$, respectively; log-rank test).

doi:10.1371/journal.pone.0101118.g002

Madison, WI), as described elsewhere [28,29]. HeLa cells were seeded in 48-well culture plates and cultured at $37^{\circ} \mathrm{C}$ in $5 \% \mathrm{CO}_{2}$. After incubation for $24 \mathrm{~h}$, the cells were washed twice with prewarmed serum-free Dulbecco's modified Eagle's medium (DMEM). Overnight-cultured $V$. vulnificus bacteria were incubated in fresh $2.5 \% \mathrm{NaCl}$ heart infusion (HI) broth for $3 \mathrm{~h}$. The logarithmically growing culture was harvested by centrifugation, washed with PBS, and re-suspended in PBS. Then, $2 \times 10^{5} \mathrm{HeLa}$ cells $/ \mathrm{mL}$ in $250 \mu \mathrm{L}$ of DMEM were infected with $5 \times 10^{5} \mathrm{cfu} / \mathrm{mL}$ V. vulnificus with or without $1 / 4$ MICs of antibiotics for $120 \mathrm{~min}$. Lactate dehydrogenase (LDH) released into the supernatant fluid was assayed as a marker of cytotoxicity, in accordance with the manufacturer's protocol.

\section{Effects of sub-inhibitory concentrations of antibiotics on rtxA1 expression}

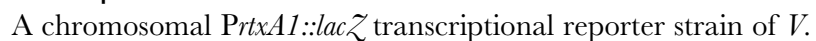
vulnificus MO6-24/O was constructed as described previously $[29,30]$. An overnight culture of the reporter strain was inoculated in $2.5 \% \mathrm{NaCl} \mathrm{HI}$ broth at a concentration of $5 \times 10^{5} \mathrm{cfu} / \mathrm{mL}$ with or without $1 / 4 \mathrm{MICs}$ of antibiotics for $120 \mathrm{~min}$. The culture was lysed in lysis buffer, and $\beta$-galactosidase activity was assayed using $2 \times \beta$-galactosidase substrate $(0.2 \mathrm{M}$ sodium phosphate buffer, $2 \mathrm{mM} \quad \mathrm{MgCl}_{2}, 100 \mathrm{mM}$ mercaptoethanol, $1.33 \mathrm{mg} / \mathrm{mL}$ Onitrophenyl- $\beta$-D-galactopyranoside), following the method described previously [30]. All experiments were performed three or more times.

\section{Statistical analyses}

The Kolmogorov-Smirnov goodness-of-fit test was used to determine the distribution of each set of data for normality before analysis, and continuous variables were compared using Student's $t$-test. Survival analysis was performed using the Kaplan-Meier method and log-rank test. All tests of significance were two-tailed, and $P$-values $\leq 0.05$ were deemed to indicate statistical significance. Statistical analyses of the data were performed using the SPSS ver. 19.0 software (SPSS, Chicago, IL) and the GraphPad Prism ver. 5.0 program (GraphPad Software, La Jolla, CA).

\section{Results}

In vitro time-kill assay

The MICs of cefotaxime, minocycline, and ciprofloxacin for $V$. vulnificus CMCP6 were 0.0625, 0.0625, and $0.03 \mathrm{mg} / \mathrm{L}$, respectively. In the time-kill assay using 3/4 MICs of each antibiotic, the cefotaxime-ciprofloxacin combination was found to have synergistic activity against $V$. vulnificus CMCP6, and the bacterial colony counts after $24 \mathrm{~h}$ of in vitro treatment were lower for cefotaximeplus-ciprofloxacin than for cefotaxime, ciprofloxacin, minocycline or cefotaxime-plus-minocycline (Fig. 1).

\section{Survival rates of mice with $V$. vulnificus sepsis treated with various antibiotic regimens}

Figure 2 presents the survival rates of each treatment group after inoculation with $1 \times 10^{8} \mathrm{cfu} V$. vulnificus CMCP6. All 10 control mice died within $12 \mathrm{~h}$. The 96-h survival rate was significantly higher in the cefotaxime-plus-ciprofloxacin group $(85 \%, 17 / 20)$ compared with the cefotaxime $(0 \%, 0 / 20)$ and cefotaxime-plus-minocycline groups $(35 \%, 7 / 20) \quad(P<0.001$, $P=0.003$, respectively). The 96 -h survival rate in the cefotaxime-plus-ciprofloxacin group was also higher than that in the ciprofloxacin group $(65 \%, 13 / 20)$, although the difference was not statistically significant $(P=0.148)$.

In vivo clearance of $V$. vulnificus from mice treated with various antibiotic regimens

In experiments where $1 \times 10^{7} \mathrm{cfu} V$. vulnificus CMCP6 was used as the initial inoculum, all infected control mice $(n=6)$ died within $12 \mathrm{~h}$, whereas all 48 of the infected mice treated with cefotaxime $(\mathrm{n}=12)$, ciprofloxacin $(\mathrm{n}=12)$, cefotaxime-plus-ciprofloxacin $(\mathrm{n}=12)$, or cefotaxime-plus-minocycline $(\mathrm{n}=12)$ were still alive after $96 \mathrm{~h}$.

The viable bacterial counts in liver were lower in mice treated with cefotaxime-plus-ciprofloxacin than in those treated with cefotaxime alone $(P<0.001$ at $24 \mathrm{~h}$ and $48 \mathrm{~h}$, each), ciprofloxacin alone $(P=0.030$ at $24 \mathrm{~h} ; P=0.001$ at $48 \mathrm{~h})$ and cefotaxime-plusminocycline $(P=0.044$ at $24 \mathrm{~h} ; P=0.008$ at $48 \mathrm{~h})$. The viable bacterial counts in spleen were lower in mice treated with cefotaxime-plus-ciprofloxacin than those treated with cefotaxime alone $(P<0.001)$ and ciprofloxacin alone $(P=0.003)$ at $24 \mathrm{~h}$ and those treated with cefotaxime alone $(P<0.001)$ and cefotaxime- 


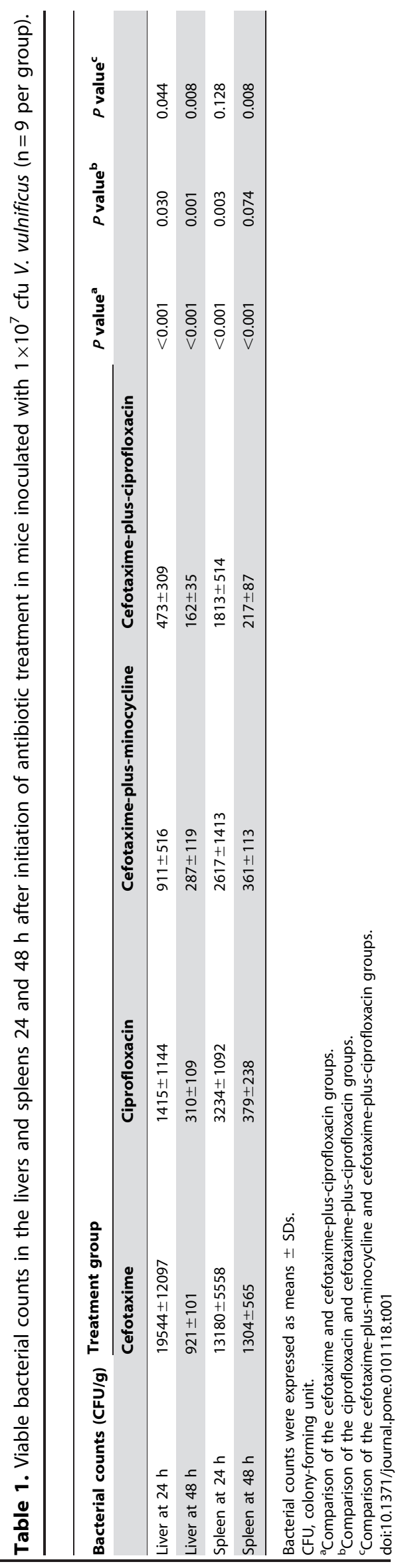

PLOS ONE | www.plosone.org plus-minocycline $(P=0.008)$ at $48 \mathrm{~h}(\mathrm{n}=9$ mice per group; Table 1).

\section{In vitro effect of sub-inhibitory concentrations of antibiotics on RtxA1-mediated cytotoxicity and the transcription of $r$ txA1}

The MICs of cefotaxime, minocycline, and ciprofloxacin for $V$. vulnificus MO6-24/O were $0.0625,0.0625$, and $0.03 \mathrm{mg} / \mathrm{L}$, respectively. The impaired cytotoxicity of CMM770 ( $\Delta r t x A 1)$, as compared with MO6-24/O (WT), indicates that the cytotoxicity noted at 120 min was mainly due to RtxAl (Fig. 3A). V. vulnificusinduced cytotoxicity was inhibited more effectively by $1 / 4 \mathrm{MIC}$ of ciprofloxacin than by $1 / 4 \mathrm{MIC}$ of cefotaxime or $1 / 4 \mathrm{MIC}$ of minocycline $(P<0.05$ for each). Similarly, the transcription of $r t x A 1$ was inhibited more efficiently by $1 / 4 \mathrm{MIC}$ of ciprofloxacin than by $1 / 4 \mathrm{MIC}$ of cefotaxime or $1 / 4 \mathrm{MIC}$ of minocycline $(P<0.05$ for each; Fig. 3B).

\section{Discussion}

A previous in vitro study demonstrated a synergistic bactericidal effect of cefotaxime-plus-ciprofloxacin against $V$. vulnificus ATCG 27562 [22]. In the present study, we demonstrated that the combination of ciprofloxacin-plus-cefotaxime acts synergistically, and exhibits more potent bactericidal activity in vitro against $V$. vulnificus CMCP6 than either cefotaxime-plus-minocycline or ciprofloxacin alone. In vitro synergism for the combination of cefotaxime-plus-ciprofloxacin against enteric Gram-negative pathogens also has been reported for E. coli [19], Serratia marcescens [31], Pseudomonas aeruginosa [32], and Salmonella enterica serotypes Typhi and Paratyphi [20,21,33]. One possible mechanism underlying this synergism may be the interaction of quinolones with the outer membrane, with the quinolones acting as chelating agents, thereby increasing its permeability to $\beta$-lactam antibiotics [19].

We performed a survival analysis using $10^{8} \mathrm{cfu} V$. vulnificus as the initial inoculum, as a previous study showed in vivo synergism for cefotaxime and minocycline [14]. In that study, the mortality rates of cefotaxime-treated and cefotaxime-plus-minocycline-treated mice were $0 \%$ and $40 \%$, respectively, similar to our results of $0 \%$ and $35 \%$, respectively. However, the efficacy of quinolones was not directly compared with the other regimens using a high inoculum $\left(10^{8} \mathrm{cfu}\right)$, as a subsequent study using quinolones [13] was performed with a lower inoculum $\left(1.5 \times 10^{7} \mathrm{cfu}\right)$ of $V$. vulnificus. Here, the survival rate was higher among mice treated with ciprofloxacin-based regimens than among mice treated with the cefotaxime-minocycline combination, although the in vivo $V$. vulnificus clearance rates for ciprofloxacin monotherapy and cefotaxime-plus-minocycline therapy were similar $(P=0.25,0.32$, $0.68,0.84$ in liver at $24 \mathrm{~h}$, spleen at $24 \mathrm{~h}$, liver at $48 \mathrm{~h}$, spleen at $48 \mathrm{~h})$.

To compare the efficacies of the treatment regimens on host survival and bacterial numbers in the organs, we infected mice with $1 \times 10^{7}$ cfu $V$. vulnificus. Although all infected control mice died rapidly, all of the mice in the treatment groups survived, regardless of the regimen used, including cefotaxime monotherapy. Similar results were reported by Tang et al. [13], who compared the efficacies of fluoroquinolone and other agents using $1.5 \times 10^{7} \mathrm{cfu} V$. vulnificus; they found that the survival rates of the mice were excellent at $>80 \%$, regardless of the administered regimen. Kim et al. [30] performed a survival analysis after antibiotic therapy using $10^{8} \mathrm{cfu} V$. vulnificus as the initial inoculum [34], based on the finding that none of the mice that were inoculated with $10^{7} \mathrm{cfu} V$. vulnificus and treated with antibiotics died, regardless of the regimen (personal communication). We considered it unfeasible to 
A.

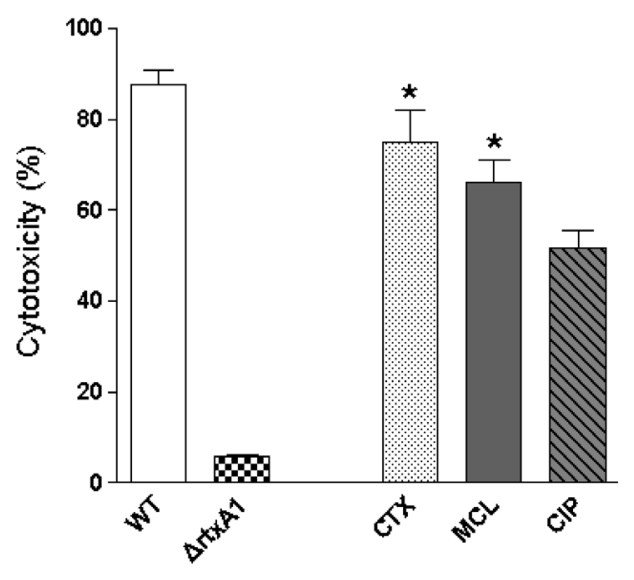

B.

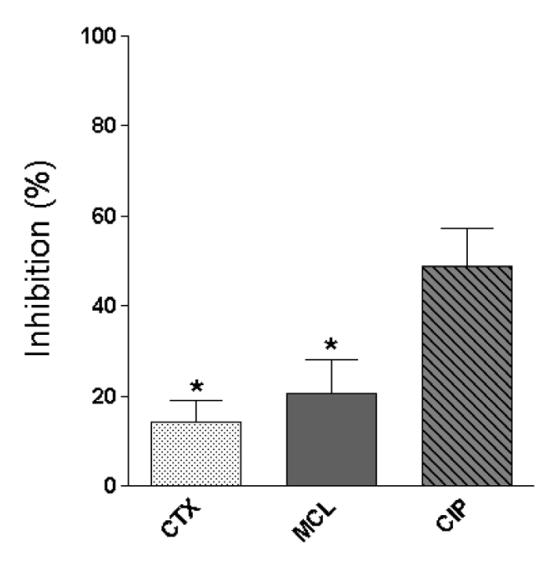

PrtxA1::lacZ reporter

Figure 3. The effects of sub-inhibitory concentrations of antibiotics on $\mathrm{V}$. vulnificus cytotoxicity and rtxA 1 transcription. A. Cytotoxicity assay. The impaired cytotoxicity of $\triangle r t x A 1$ compared with that of the WT strain shows that cytotoxicity at 120 min is due principally to RtxA1. $V$. vulnificus cytotoxicity is inhibited more markedly by $1 / 4$ MIC of ciprofloxacin than by $1 / 4$ MICs of cefotaxime or minocycline ( $n=12$ per group). B. Transcriptional reporter assay. The transcription of $r$ txA 1 is more efficiently inhibited by $1 / 4 \mathrm{MIC}$ of ciprofloxacin than by $1 / 4 \mathrm{MICs}$ of cefotaxime or minocycline ( $\mathrm{n}=4$ per group). WT, MO6-24/O; $\triangle r t x A 1$, CMM770 (MO6-24/O background with a deletion mutation in the $r t x A 1$ gene); CTX, cefotaxime; CIP, ciprofloxacin; MCL, minocycline. ${ }^{*} P<0.05$ compared to the values for ciprofloxacin (Student's $t$-test). doi:10.1371/journal.pone.0101118.g003

compare the survival rates for different antibiotic regimens when $<10^{8} \mathrm{cfu} / \mathrm{mL}$. vulnificus were used as the initial inoculum, given that many samples would be needed to show a statistically significant difference if the survival rate was $>80 \%$ for all regimens used. Therefore, we enumerated the bacteria in the organs of the inoculated and treated mice and found that cefotaxime-ciprofloxacin was the most effective regimen in terms of clearing $V$. vulnificus. These data provide further evidence for the superior efficacy of cefotaxime-plus-ciprofloxacin regimens in clearing $V$. vulnificus in vivo.

Sub-inhibitory concentrations of antibiotics interfere with the processes of host-parasite interactions, such as phagocytosis, adherence, and toxin production. For this reason, the anti-toxin effects of sub-inhibitory levels of antibiotics have been studied specifically in pathogens that cause rapidly fatal toxin-related diseases, such as necrotizing skin and soft tissue infections, in which anti-toxin efficacy is therapeutically important [35-38]. In our previous studies, we showed that RtxAl is a major virulence factor of $V$. vulnificus [3,7], and that deletion of $r t x A 1$ or its regulators decrease the cytotoxicity and increase the $\mathrm{LD}_{50}$ significantly in mice $[6,25,28,30,39]$. We also showed that RtxAl can be suppressed by certain compounds [29,40] and subinhibitory concentrations of chloramphenicol [25]. However, although RtxAl is known as a major virulence element in $V$. vulnificus, and $V$. vulnificus causes rapidly fatal necrotizing skin and soft tissue infections, the effects of sub-inhibitory concentration of antibiotics that are frequently used in therapy on RtxAl production have not been evaluated to date. In the present study, we showed that ciprofloxacin more effectively suppressed $r t x A 1$ transcription and protected host cells than either minocycline or cefotaxime. In a previous study, suppressing RtxAl production per se without direct killing of bacteria was shown to reduce the mortality rate of mice infected with $V$. vulnificus [29]. The more potent inhibitory effect of ciprofloxacin on RtxAl-induced cytotoxicity may explain the improved survival of mice treated with ciprofloxacin-based regimens, compared with mice treated with regimens using cefotaxime or doxycycline.

Certain conclusions that we can draw from this study are limited, as we examined only the combination of ciprofloxacinplus-cefotaxime. Further studies will be needed to evaluate the efficacy of combinations of ciprofloxacin or minocycline and other third-generation cephalosporins, including ceftazidime and cefrtriaxone.

In summary, we found that, at sub-inhibitory concentrations, ciprofloxacin is more effective at reducing $r t x A 1$ transcription and subsequent cytotoxicity than either minocycline or cefotaxime. Moreover, we demonstrated that the combination of ciprofloxacin and cefotaxime is more effective in clearing $V$. vulnificus in vivo than commonly used regimens, which suggests that this combination is a good candidate for the treatment of $V$. vulnificus sepsis in humans.

\section{Acknowledgments}

Presented in part: 52nd Annual Interscience Conference on Antimicrobial Agents and Chemotherapy (ICAAC), San Francisco, September 9-12, 2012 (abstract no. B-1298).

\section{Author Contributions}

Conceived and designed the experiments: HCJ. Performed the experiments: HCJ SMC HKK SEK. Analyzed the data: HCJ. Contributed reagents/materials/analysis tools: SJK KHP PYR THL YRK JHR HEC. Wrote the paper: HCJ SIJ HEG. 


\section{References}

1. Dechet AM, Yu PA, Koram N, Painter J (2008) Nonfoodborne Vibrio infections: an important cause of morbidity and mortality in the United States, 1997-2006. Clin Infect Dis 46: 970-976.

2. Klontz KC, Lieb S, Schreiber M, Janowski HT, Baldy LM, et al. (1988) Syndromes of Vibrio vulnificus infections. Clinical and epidemiologic features in Florida cases, 1981-1987. Ann Intern Med 109: 318-323.

3. Chung KJ, Cho EJ, Kim MK, Kim YR, Kim SH, et al. (2010) RtxAl-induced expression of the small GTPase Rac2 plays a key role in the pathogenicity of Vibrio vulnificus. J Infect Dis 201: 97-105.

4. Kwak JS, Jeong HG, Satchell KJ (2011) Vibrio vulnificus rtxAl gene recombination generates toxin variants with altered potency during intestinal infection. Proc Natl Acad Sci U S A 108: 1645-1650.

5. Lo HR, Lin JH, Chen YH, Chen CL, Shao CP, et al. (2011) RTX toxin enhances the survival of Vibrio vulnificus during infection by protecting the organism from phagocytosis. J Infect Dis 203: 1866-1874.

6. Jeong KC, Jeong HS, Rhee JH, Lee SE, Chung SS, et al. (2000) Construction and phenotypic evaluation of a Vibrio vulnificus vvpE mutant for elastolytic protease. Infect Immun 68: 5096-5106.

7. Kim YR, Lee SE, Kang IC, Nam KI, Choy HE, et al. (2013) A Bacterial RTX Toxin Causes Programmed Necrotic Cell Death Through Calcium-Mediated Mitochondrial Dysfunction. J Infect Dis.

8. Yokochi N, Tanaka S, Matsumoto K, Oishi H, Tashiro Y, et al. (2013) Distribution of virulence markers among Vibrio vulnificus isolates of clinical and environmental origin and regional characteristics in Japan. PLoS One 8: e55219.

9. Morrison SS, Williams T, Gain A, Froelich B, Taylor C, et al. (2012) Pyrosequencing-based comparative genome analysis of Vibrio vulnificus environmental isolates. PLoS One 7: e37553.

10. Sugiyama H, Kashimoto T, Ueno S, Ehara H, Kodama T, et al. (2011) Relationship between localization on cellular membranes and cytotoxicity of Vibrio vulnificus hemolysin. PLoS One 6: e26018.

11. Chen SC, Chan KS, Chao WN, Wang PH, Lin DB, et al. (2010) Clinical outcomes and prognostic factors for patients with Vibrio vulnificus infections requiring intensive care: a 10-yr retrospective study. Crit Care Med 38: 1984 1990.

12. Chuang YC, Liu JW, Ko WC, Lin KY, Wu JJ, et al. (1997) In vitro synergism between cefotaxime and minocycline against Vibrio vulnificus. Antimicrob Agents Chemother 41: 2214-2217.

13. Tang HJ, Chang MC, Ko WC, Huang KY, Lee CL, et al. (2002) In vitro and in vivo activities of newer fluoroquinolones against Vibrio vulnificus. Antimicrob Agents Chemother 46: 3580-3584.

14. Chuang YC, Ko WC, Wang ST, Liu JW, Kuo CF, et al. (1998) Minocycline and cefotaxime in the treatment of experimental murine Vibrio vulnificus infection. Antimicrob Agents Chemother 42: 1319-1322.

15. Liu JW, Lee IK, Tang HJ, Ko WC, Lee HC, et al. (2006) Prognostic factors and antibiotics in Vibrio vulnificus septicemia. Arch Intern Med 166: 2117-2123.

16. Chen SC, Lee YT, Tsai SJ, Chan KS, Chao WN, et al. (2012) Antibiotic therapy for necrotizing fasciitis caused by Vibrio vulnificus: retrospective analysis of an 8 year period. J Antimicrob Chemother 67: 488-493.

17. Kim DM, Cho HS, Kang JI, Kim HS, Park CY (2008) Deferasirox plus ciprofloxacin combination therapy after rapid diagnosis of Vibrio vulnificus sepsis using real-time polymerase chain reaction. J Infect 57: 489-492.

18. Kim CM, Park RY, Choi MH, Sun HY, Shin SH (2007) Ferrophilic characteristics of Vibrio vulnificus and potential usefulness of iron chelation therapy. J Infect Dis 195: 90-98.

19. Chapman JS, Georgopapadakou NH (1988) Routes of quinolone permeation in Escherichia coli. Antimicrob Agents Chemother 32: 438-442.

20. Neupane GP, Kim DM, Kim SH, Lee BK (2010) In vitro synergism of ciprofloxacin and cefotaxime against nalidixic acid-resistant Salmonella enterica serotypes Paratyphi A and Paratyphi B. Antimicrob Agents Chemother 54: 3696-3701.
21. Kim DM, Neupane GP, Jang SJ, Kim SH, Lee BK (2010) In vitro efficacy of the combination of ciprofloxacin and cefotaxime against nalidixic acid-resistant Salmonella enterica serotype Typhi. Int J Antimicrob Agents 36: 155-158.

22. Kim DM, Lym Y, Jang SJ, Han H, Kim YG, et al. (2005) In vitro efficacy of the combination of ciprofloxacin and cefotaxime against Vibrio vulnificus. Antimicrob Agents Chemother 49: 3489-3491.

23. Korea Food and Drug Administration (2011) Guidelines for animal experiments and IACUC standard opreation. Available: http://www.mfds.go.kr/labanimal/ index.do? mid $=56 \&$ seq $=11795 \& \mathrm{cmd}=\mathrm{v}$. Accessed 7 June 2014 .

24. Kim HU, Kim SY, Jeong H, Kim TY, Kim JJ, et al. (2011) Integrative genomescale metabolic analysis of Vibrio vulnificus for drug targeting and discovery. Mol Syst Biol 7: 460.

25. Kim YR, Lee SE, Kook H, Yeom JA, Na HS, et al. (2008) Vibrio vulnificus RTX toxin kills host cells only after contact of the bacteria with host cells. Cell Microbiol 10: 848-862.

26. Clinical and Laboratory Standards Institute (2009) Methods for dilution antimicrobial susceptibility tests for bacteria that grow aerobically; approved standard-eighth edition. Wayne: Clinical and Laboratory Standards Institute. M07-A8.

27. Lee SE, Kim SY, Kim CM, Kim MK, Kim YR, et al. (2007) The pyrH gene of Vibrio vulnificus is an essential in vivo survival factor. Infect Immun 75: 2795 2801.

28. Kim YR, Lee SE, Kim CM, Kim SY, Shin EK, et al. (2003) Characterization and pathogenic significance of Vibrio vulnificus antigens preferentially expressed in septicemic patients. Infect Immun 71: 5461-5471.

29. Kim JR, Cha MH, Oh DR, Oh WK, Rhee JH, et al. (2010) Resveratrol modulates RTX toxin-induced cytotoxicity through interference in adhesion and toxin production. Eur J Pharmacol 642: 163-168.

30. Kim SY, Lee SE, Kim YR, Kim CM, Ryu PY, et al. (2003) Regulation of Vibrio vulnificus virulence by the LuxS quorum-sensing system. Mol Microbiol 48: $1647-1664$.

31. Otsuki M, Nishino T (1996) The synergic effects of quinolones and oral cephem antibiotics on Serratia marcescens. J Antimicrob Chemother 38: 771-776.

32. Piccoli L, Guerrini M, Felici A, Marchetti F (2005) In vitro and in vivo synergy of levofloxacin or amikacin both in combination with ceftazidime against clinical isolates of Pseudomonas aeruginosa. J Chemother 17: 355-360.

33. Chang CM, Chuang YC, Wu JJ, Wang LR, Lee HC, et al (2009) In vitro combination of cefotaxime and ciprofloxacin against non-typhoid salmonellae. Int J Antimicrob Agents 33: 593-594

34. Neupane GP, Kim DM, Yun NR, Shin SH, Lim SC, et al. (2012) Quantitative PCR and in vivo efficacy of antibiotics in the treatment of Vibrio vulnificus infection in a mouse model. Eur J Clin Microbiol Infect Dis 31: 2461-2467.

35. Gemmell CG, Ford CW (2002) Virulence factor expression by Gram-positive cocci exposed to subinhibitory concentrations of linezolid. J Antimicrob Chemother 50: 665-672.

36. Dumitrescu O, Boisset S, Badiou C, Bes M, Benito Y, et al. (2007) Effect of antibiotics on Staphylococcus aureus producing Panton-Valentine leukocidin. Antimicrob Agents Chemother 51: 1515-1519.

37. Ohlsen K, Ziebuhr W, Koller KP, Hell W, Wichelhaus TA, et al. (1998) Effects of subinhibitory concentrations of antibiotics on alpha-toxin (hla) gene expression of methicillin-sensitive and methicillin-resistant Staphylococcus aureus isolates. Antimicrob Agents Chemother 42: 2817-2823.

38. Saleh-Mghir A, Dumitrescu O, Dinh A, Boutrad Y, Massias L, et al. (2012) Ceftobiprole efficacy in vitro against Panton-Valentine leukocidin production and in vivo against community-associated methicillin-resistant Staphylococcus aureus osteomyelitis in rabbits. Antimicrob Agents Chemother 56: 6291-6297.

39. Lee SE, Ryu PY, Kim SY, Kim YR, Koh JT, et al. (2004) Production of Vibrio vulnificus hemolysin in vivo and its pathogenic significance. Biochem Biophys Res Commun 324: 86-91.

40. Na HS, Cha MH, Oh DR, Cho CW, Rhee JH, et al. (2011) Protective mechanism of curcumin against Vibrio vulnificus infection. FEMS Immunol Med Microbiol 63: 355-362. 